\title{
Mirada de la comunidad educativa acerca del desempeño de directivas educativas: Algunas reflexiones
}

\section{Perspective of the Educational Community about the Performance of School Directors: Some Reflections}

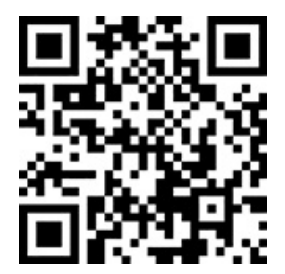

\author{
Ileana Vargas-Jiménez ${ }^{1}$ \\ Universidad Nacional \\ Consejo Universitario \\ Heredia, Costa Rica \\ ileana.vargas.jimenez@una.cr \\ http://orcid.org/0000-0003-2570-6862
}

Recibido 16 de junio de 2016 • Corregido 21 de noviembre de 2016 • Aceptado 3 de diciembre de 2016

\begin{abstract}
Resumen: Se seleccionaron dos instituciones educativas con el objetivo de caracterizar el desempeño de dos directoras desde la mirada de la comunidad educativa: docentes, estudiantes, asistente administrativa y, padres y madres de familia. Para llevar a cabo lo anterior fue necesario plantearse: ¿cómo valoran los miembros de la comunidad educativa de dos centros educativos el desempeño profesional, en la práctica directiva de dos directoras graduadas del Centro de Investigación y Docencia en Educación (CIDE) de la Universidad Nacional, con respecto al ejercicio de liderazgo, procesos de comunicación y toma de decisiones? Se trabajó con 28 participantes, las entrevistas y los grupos focales, luego se procedió a utilizar la herramienta de ATLAS TI para interpretar las categorías de análisis. Como conclusiones principales se destacan que una gestión de calidad debe ser un proceso de participación, trabajo en equipo, comunicación y acercamiento desde la dirección con las personas involucradas. Asimismo, se concibe que un buen desempeño es aquel para quien el estudiantado es prioridad, ayuda a las demás personas con humildad, cariño, sabe escuchar, brinda confianza, es negociador, y sabe delegar.
\end{abstract}

Palabras claves: Investigación cualitativa, liderazgo, comunicación, toma de decisiones.

\footnotetext{
1 Doctora en Educación por la Universidad Estatal a Distancia, Costa Rica, Máster en Administración Educativa por la Universidad de New México, Estados Unidos. Investigadora y profesora en la División de Educación para el Trabajo. Fue directora de la División de Educación para el Trabajo del Centro de Investigación y Docencia en Educación. Exdecana del Centro de Investigación y Docencia en Educación. Actualmente es representante del sector académico en el Consejo Universitario de la Universidad Nacional. Posee varias publicaciones entre libros y artículos de revistas científicas.
} 
doi: http://dx.doi.org/10.15359/ree.21-1.14

URL: http://www.una.ac.cr/educare

CORREO: educare@una.cr

\begin{abstract}
Two educational institutions were selected with the aim of characterizing the performance of two principals from the perspective of the educational community: teachers, students, administrative assistant and parents. In order to carry out the study, it was necessary to consider how the members of the educational community of two schools evaluate the professional performance related to the exercise of leadership, communication processes and decision making, during the internship of two CIDE graduates of the UNA. We worked the interviews and the focus groups with twenty-eight participants; later, we used the ATLAS TI tool to interpret the analysis categories. The main conclusions are that a quality management must be a process of participation, teamwork, communication and approach from the school administration to the people involved. Likewise, it is conceived that a good performance means to consider students as a priority, to help others with humility and affection, to know how to listen, to provide confidence; and that the principal considers students as a priority, and is a negotiating person who knows how to delegate. In short, a good principal is the person who can lead an institution by exercising good leadership, and, therefore, will influence the proper management of communication and decision-making.
\end{abstract}

Keywords: Qualitative research, leadership, communication, making-decision.

\title{
Introducción
}

Resulta sorprendente que la bibliografía científica y las regulaciones administrativas demuestran que se están consolidando nuevos modelos de organización y gestión de centros educativos, los cuales exigen, rotundamente, la presencia y la actuación de una dirección diferenciada como eje central sobre el que se reestructura la vida del centro escolar. $Y$ eso es respaldado por el reciente informe 2021 metas educativas. La educación que queremos para la generación de los bicentenarios (Organización de Estados Iberoamericanos para la Educación, la Ciencia y la Cultura, OEl, 2010) al plantear que la gestión escolar debe implicar una diferencia de enfoque con respecto a la administración educativa, y que esta debe ir acompañada de la participación de la comunidad educativa donde la gestión escolar se comprende desde tres ejes:

1. Los procesos de enseñanza y aprendizaje

2. Las relaciones entre los diferentes actores

3. La estructura y funcionamiento

Para Coronel (2005), el liderazgo del profesorado es la representación de una forma diferente de concebir y relacionarse con el centro educativo, ya que afecta el concepto de la enseñanza como profesión. El liderazgo es socialmente construido y se inserta en la estructura de la cultura organizacional. Por ello, se elabora un aprendizaje mutuo, donde se comparten percepciones, valores, opiniones e informaciones a través de la conversación. 
El desarrollo del liderazgo dentro de las instituciones escolares conlleva un cambio dentro de la organización escolar, principalmente, de quienes ejercen la dirección con el encargo de gestionar, liderar y de coadyuvar los procesos administrativos. Se debe procurar mantener un clima escolar apropiado para el cambio y los procesos que se desarrollan en la institución, para lo cual se requiere de una adecuada inteligencia emocional para conocer al personal docente que está a su cargo.

El centro educativo, y particularmente quien está en la gestión, es el responsable directo del trabajo que se lleva a cabo en el plantel, de su persona dependen los procesos y los resultados, hoy más que nunca, el centro educativo debe responder con procesos inclusivos y con programas de calidad y, de no prestarse atención a la problemática, se continuará con resultados lamentables especialmente para las escuelas públicas de Costa Rica y para la formación de personal administrador educativo desde las universidades, pues constituye esta una relación causal, según Cano (2009) y Vargas (2010).

De acuerdo con Cano (2009), las tendencias mundiales señalan que la preparación profesional del personal gestor educativo es esencial para el logro de los objetivos institucionales. Además, el centro educativo es la institución por excelencia donde debe centrarse el cambio y la mejora institucional y eso es coincidente con varias investigaciones (Barrera-Osorio, Fasih, Patrinos y Santibáñez, 2009; Concha, 2007; OEl, 2010).

Es asícomo interesa caracterizar el desempeño de dos directoras en el contexto educativo desde la mirada de los diferentes actores involucrados en el proceso. Para llevar a cabo lo anterior se plantea la siguiente interrogante: ¿Cómo valoran los miembros de la comunidad educativa de dos centros educativos el desempeño profesional, en la práctica directiva de dos directoras graduadas del CIDE de la UNA, con respecto al ejercicio de liderazgo, procesos de comunicación y toma de decisiones?

\section{Marco conceptual}

En el contexto de esta investigación, la práctica directiva es concebida como"la conjunción de percepciones, creencias, y formas de pensar, en que se lleva a cabo el trabajo diario en la institución escolar" (Latorre, 2006). Esta noción implica reconocer, por parte de las directoras y luego por los miembros de la comunidad educativa de un cuerpo de conocimientos, saberes y creencias que sustentan y orientan las decisiones y acciones del personal responsable de dirigir un centro educativo. Por lo que la formación profesional recibida es también importante para el ejercicio de la práctica directiva (Cuenca, 2012).

Garbanzo y Orozco (2010) explican que, para que se realice de forma asertiva el desarrollo de las organizaciones educativas, es requerida la participación de un liderazgo renovador, con el cual se pretende llevar a cabo una modificación de los modelos. Estos modelos con mayor autonomía institucional y gestión moderna de procesos en cada contexto específico permiten 
doi: http://dx.doi.org/10.15359/ree.21-1.14

URL: http://www.una.ac.cr/educare

CORREO: educare@una.cr

no solo que haya un mejoramiento en las ofertas académicas, sino también, una mayor posibilidad de organización en las tareas y el potencial humano necesario.

Se puede decir que el trabajo en las organizaciones educativas debe ser de carácter colaborativo, ya que esto permite un aprendizaje recíproco entre sus miembros $y$, a la vez, que las limitaciones existentes sean suplidas por la cooperación de sus integrantes. Este proceso permite que las organizaciones educativas sean eficientes, no solo en el ámbito académico, al desarrollar las capacidades cognitivas, sino que propicia un desarrollo en la formación integral, donde la ética, estética y el componente cultural y espiritual se implementan de manera responsable.

Es posible confirmar que el liderazgo en el ejercicio de los responsables de dirigir instituciones educativas es decisivo para el mejoramiento de la calidad de la educación y, por ende, ha cobrado relevancia en el debate pedagógico, la Figura 1 resume la concepción de liderazgo del director y la directora.

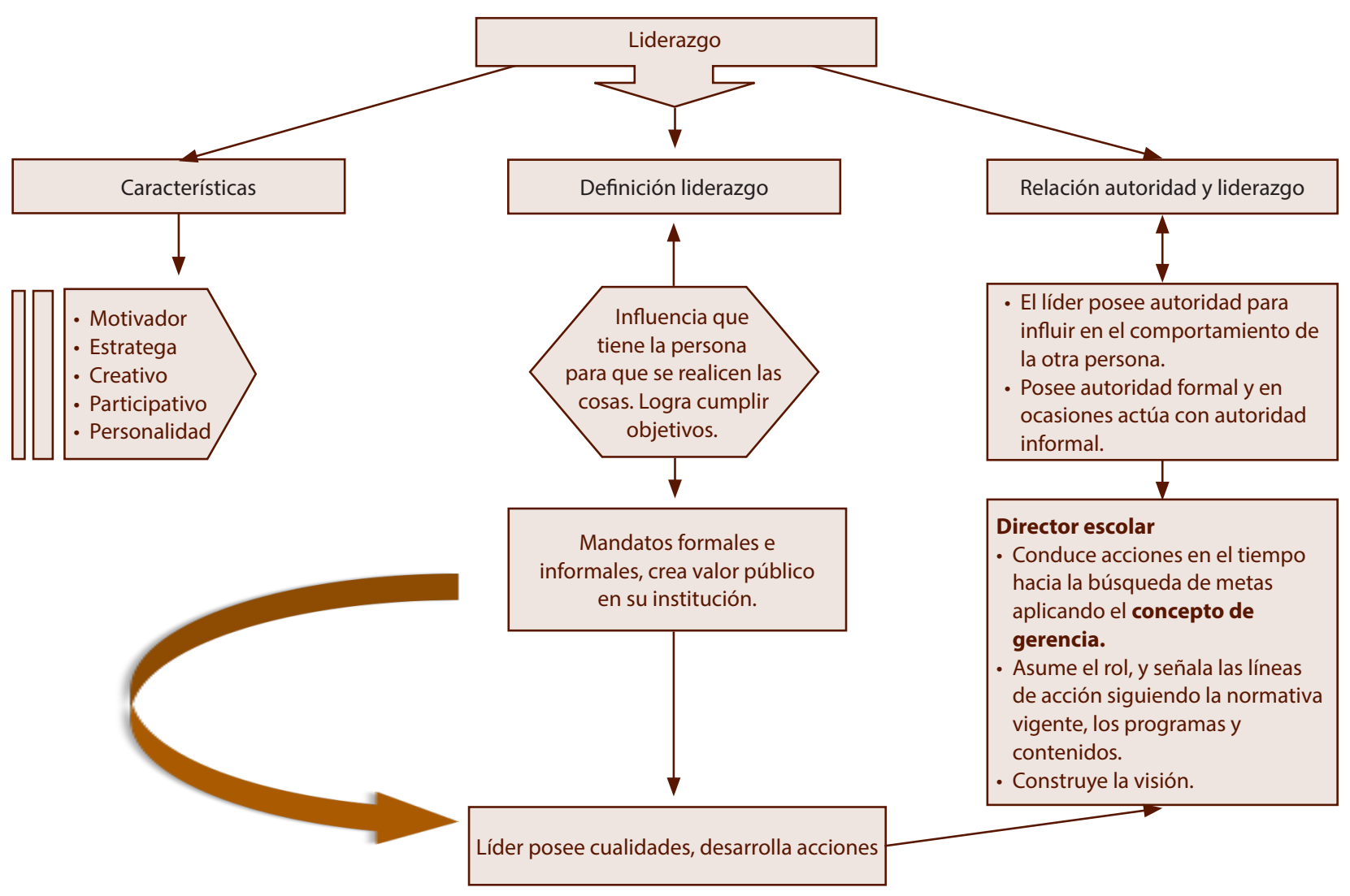

Figura 1. Resume la concepción de liderazgo del personal encargado de dirigir la institución educativa Vargas (2007). 
doi: http://dx.doi.org/10.15359/ree.21-1.14

URL: http://www.una.ac.cr/educare

CORREO: educare@una.cr

El desarrollo del liderazgo dentro de las instituciones escolares conlleva un cambio dentro de la organización escolar, principalmente, en gestionar, liderary coadyuvar los procesos administrativos. Se debe procurar mantener un clima escolar apropiado para el cambio y los procesos que se desarrollan en la institución, para lo cual se requiere una adecuada inteligencia emocional del personal directivo que debe conocer al personal docente que está a su cargo. La comunicación es un aspecto de vital trascendencia en la organización escolar y es crucial para el éxito de la institución, en general, debido a que, en las relaciones interpersonales desarrolladas dentro de la organización, la comunicación permite a sus miembros intercambiar información, trabajo, procedimientos, entre otros aspectos.

La comunicación debe ser vista como una herramienta poderosa para el éxito y el crecimiento de las personas que integran la organización. Hay que mencionar lo que Pareja (2009) explica en cuanto a que la comunicación es un intercambio, ya que un miembro del grupo transmite por medio de un mensaje (persona emisora), el cual llega a ser recibido (persona receptora) y respondido.

Por un lado, Oliver (2001, citado por Pareja, 2009), describe diferentes conflictos que se pueden desarrollar debido a las interferencias en la comunicación:

- Interferencias en la persona emisora: la intencionalidad de quien emite el mensaje.

- Interferencias en el mensaje: la veracidad del contenido y el contenido en sí del mensaje emitido puede acarrear, en ocasiones, conflictos.

- Interferencias en el canal: el medio por el cual se envía el mensaje puede determinar su correcta o incorrecta recepción.

- Interferencias en el código: al no manejarse un sistema lingüístico similar puede ocasionar conflictos y, también, el empleo de diferentes registros.

No cabe duda de que el centro escolar es una organización compleja en la que circula gran cantidad de información, por lo que es recomendable establecer ciertos juicios que permitan contar con los criterios necesarios en el momento oportuno. Para lograr lo anterior se requiere, según Borrel (1996, citado por Braslavsky, Acosta y Jabif, 2004), presentarla en forma concreta y clara, dar a conocer si esta afecta a los diversos actores, seleccionar un sistema ágil y eficaz para transmitirla, establecer canales claros de comunicación para que esta circule.

Igualmente, uno de los aspectos que caracteriza un centro escolar es lograr buenos sistemas de comunicación que permitan contar con suficiente información para detectar y comprender el origen de posibles ejes de tensión y luego resolver; es así como, a partir de esta perspectiva, se consolida el movimiento de mejora escolar (De Miguel et al., citado por Braslavsky et al., 2004). Es importante, desde la institución escolar, comprender su funcionamiento e incidir en su mejora a través de mecanismos que integran a todas las personas involucradas en espacios para intercambiar información útil para la acción. 
doi: http://dx.doi.org/10.15359/ree.21-1.14

URL: http://www.una.ac.cr/educare

CORREO: educare@una.cr

Por una parte, el proceso de toma de decisiones es uno de los aspectos fundamentales dentro del proceso administrativo, ya que este requiere, según Batley (1991, citado por Pozner, 2000), de enseñanza y de pericia por parte de quien toma las decisiones. Según este autor, lo primero que se debe hacer es dominar la capacidad de diferenciar entre las decisiones directas, regulares y hasta urgentes, también las decisiones complejas ya que estas requieren de un análisis más completo. De igual manera, se recomienda dentro del proceso de toma de decisiones, en primera instancia, ordenar a quién involucrar, si se organizan grupos de discusión y para divulgar la información. Desde la organización, este proceso tiene cinco etapas que vale la pena mencionar:

1. Definir los objetivos y los propósitos de la decisión.

2. Reunir los hechos y la información necesarios para tomar la decisión.

3. Determinar los cursos de acción alternativos posibles.

4. Considerar las ventajas y las desventajas de cada alternativa y elegir la más apropiada.

5. Actuar para implementar la decisión de la mejor manera, controlar los efectos de la decisión y revisarla si fuera necesario. (Pozner, 2000, p. 10)

Un buen director o directora es aquel sujeto que logra una armonía en la institución que tiene a su cargo; para lograrlo es fundamental que posea una adecuada inteligencia emocional. La inteligencia emocional es una posibilidad para que quien administra pueda ser agente de cambio, y con una apropiada comprensión de cómo abordarlo. "Se define la inteligencia emocional como la habilidad de percibir, entender y aplicar efectivamente el poder de las emociones para inspirar confianza, iniciativa, energía, creatividad y motivación en los demás" (Copper, 2003, citado por Vargas, 2004, p. 108).

Por lo que la inteligencia emocional, bien aplicada, puede ayudar a tomar mejores decisiones, a valorar las capacidades individuales de la gente que está alrededor; es decir, los individuos, al utilizar la inteligencia emocional, no solo están beneficiando el campo laboral, sino también, se están favoreciendo en lo personal, por cuanto empiezan a fijarse más y mejor en las necesidades y sentimientos de las personas que están en su alrededor.

\section{Marco metodológico}

El artículo surge de una investigación efectuada durante los años 2012 al 2014, titulada: La formación profesional de directoras y directores graduados de la carrera de Administración Educativa del Centro de Investigación y Docencia en Educación de la Universidad Nacional: Análisis de su práctica directiva en dos instituciones educativas (Vargas, 2014) y responde a un diseño mixto, la primera fase con un enfoque cuantitativo, por lo tanto, abarcó a responsables de dirigir una

6

Ileana Vargas-Jiménez 
doi: http://dx.doi.org/10.15359/ree.21-1.14

URL: http://www.una.ac.cr/educare

CORREO: educare@una.cr

institución escolar y personal administrativo graduado del Centro de Investigación y Docencia en Educación (CIDE). Para la segunda fase, de la que trata el artículo, se elaboró un enfoque cualitativo, se trabajó con la comunidad educativa, la cual comprendía a los padres y las madres de familia, docentes, estudiantes y apoyo administrativo de dos instituciones educativas en las que ambas directoras debían ser graduadas del CIDE de la Universidad Nacional.

Esta investigación se enmarcó dentro de un paradigma cualitativo que se desarrolla a través del método fenomenológico, que se enfoca, según Mertens, citado por Hernández, Fernández y Baptista (2010), en las experiencias individuales subjetivas de los sujetos participantes. Para lograr lo anterior, se espera, desde la fenomenología, describir "las experiencias vividas por una persona o grupo de personas acerca de un concepto o fenómeno" (Creswell, 1998, citado por Sandín, 2003, p. 151). Interesa la fenomenología, por cuanto se preocupa por los aspectos esenciales de tipos de experiencia. Los planteamientos metodológicos de esta corriente adoptan múltiples formas, para ello se retomarán los siguientes aspectos procedimentales mencionados por Creswell, citado por Sandín (2003), los cuales se comparten plenamente para la presente indagación.

- El investigador necesita comprender las perspectivas filosóficas que subyacen al enfoque, especialmente estudiar cómo las personas experimentan un fenómeno. ...

- El investigador propone cuestiones de investigación que exploran el significado de la experiencia para las personas y les solicita que lo describan desde sus experiencias de vida cotidianas.

- El investigador recoge datos de las personas que han experimentado el fenómeno que se está investigando.

- Los pasos del análisis de datos fenomenológicos incluyen la identificación de dimensiones; estas son transformadas en clusters de significados, expresados en conceptos psicológicos y fenomenológicos. Finalmente, estas transformaciones llevan a una descripción general de la experiencia de lo que fue experienciado. (p. 152-153)

El propósito fundamental fue otorgar voz a las experiencias, desde las miradas de las personas involucradas, y eso es precisamente lo que se quiso llevar a cabo en la fase cualitativa con la interpretación de las voces de padres y madres de familia, docentes, apoyo administrativo y estudiantes de las dos instituciones.

\section{Selección de la muestra y entrada al campo}

La selección de los sujetos participantes, los cuales fueron 28 en total, se realizó a través de una selección intencionada, que responde a los siguientes criterios: 
doi: http://dx.doi.org/10.15359/ree.21-1.14

URL: http://www.una.ac.cr/educare

CORREO: educare@una.cr

1. Facilidad de acceso a las dos instituciones educativas elegidas para el estudio.

2. Se contactaron las dos directoras y se les explicó en qué consistía la investigación, ellas fueron las que sugirieron a los sujetos participantes, los cuales eran estudiantes de cuarto y quinto año, sus padres y madres, los profesores y profesoras en vista de la disponibilidad del horario. En la escuela la directora sugirió trabajar con estudiantes de cuarto grado, esto por la disposición de horario y docentes de estos grupos de estudiantes, así como sus papás y mamás.

3. De esta forma, se procedió a visitarlas y se les presentó el consentimiento informado, expresar en qué consistía la indagación así como el compromiso que asumía la investigadora, y la institución que participaba.

4. Para proseguir con el proceso de negociación y entrada se efectuó una reunión con los padres y madres de familia, personal docente, apoyo administrativo y estudiantado, la cual se efectuó con la finalidad de informar en qué consistió la investigación, así como solicitar de cada persona un consentimiento informado para trabajar con menores de edad.

5. Se trabajó en dos instituciones, la escuela San Juan ubicada en el centro de San Isidro de Heredia, urbana, y un colegio en Heredia, el cual ofrece una formación humanística y tiene la oferta de cuarto y quinto año solamente, el estudiantado fue de cuarto y quinto año, las madres y padres de estos y su profesorado, más adelante se presenta la Tabla 1 con la información de la población del estudio.

\section{Técnicas de recolección de la información}

La técnica que se utilizó para recoger los datos de la investigación cualitativa fue la entrevista semiestructurada y, para ello, se utilizó la entrevista individual y la entrevista grupal. Según Patton, citado por Flick (2007), una entrevista de grupo es una discusión y se realiza con un pequeño conjunto de personas sobre un tema específico, los grupos son normalmente de seis a ocho personas que participan en la entrevista durante una hora y media o dos horas.

Además, se incorporó la técnica de grupo focal. Aquí vale la pena recordar que esta es una técnica de recolección de datos, una unidad de análisis para la que, según Hernández et al. (2010), el grupo expresa y construye. Se reunió a un grupo de estudiantes para llevar a cabo el grupo focal e, igualmente, se reunió a un grupo de padres y madres de familia con la intención de trabajar los conceptos, las experiencias, emociones, creencias, opiniones acerca del rol desempeñado por la directora escolar. Se elaboraron las guías de entrevista con la ayuda de juicio de personal experto. Se elaboraron los siguientes instrumentos:

- Guía de entrevista individual a docentes

- Guía de entrevista individual a personal administrativo 
doi: http://dx.doi.org/10.15359/ree.21-1.14

URL: http://www.una.ac.cr/educare

- Guía de grupo focal a estudiantes

- Guía de grupo focal a padres y madres de familia

Además, vale la pena señalar que se llevaron a cabo preguntas abiertas para enriquecer la discusión de los miembros de la comunidad educativa. Se trabajó con grupos de discusión y, de acuerdo con Krueger (1998, citado por Gurdián, 2010, p. 208), estos "pueden ser definidos como una conversación cuidadosamente planeada, diseñada para obtener información de una [sic] área definida de interés", para el estudiantado, y padres y madres de familia.

El objeto del grupo de discusión es provocar autoconfesiones, lo cual facilita que los sujetos participantes voluntaria, transparente y libremente se retroalimenten entre sí. Asimismo, llevar a cabo la entrevista a profundidad con el cuerpo docente y la asistente administrativa de las instituciones que participaron (Ver Tabla 1).

Tabla 1: Recolección de datos de la investigación cualitativa

\begin{tabular}{|c|c|c|c|}
\hline \multicolumn{4}{|c|}{ Octubre-noviembre de 2012} \\
\hline Número de entrevistas & $\begin{array}{c}\text { Entrevista } \\
\text { semiestructurada }\end{array}$ & Grupo focal & Lugar \\
\hline Maestras (2) & $x$ & & \\
\hline Docentes 4 & $x$ & & \\
\hline Padres y madres de familia (1) escuela & & $x$ & \\
\hline Padres y madres de familia (1) Colegio & & $x$ & $\begin{array}{l}\text { Instituciones de Heredia, total } \\
\text { de personas participantes } 28\end{array}$ \\
\hline Personal administrativo (1) & $x$ & & \\
\hline Estudiantes de primaria de 4 grado (1) & & $x$ & \\
\hline Estudiantes de 4 y 5 años (1) & & $x$ & \\
\hline
\end{tabular}

Nota: Elaboración propia.

\section{Categoría de análisis de la investigación}

Para determinar las categorías de análisis del estudio, se indagaron diversos estudios (Bernardini, 2003; Coronel, 2005; Garbanzo y Orozco, 2010; Latorre, 2006; Pareja, 2009; Vargas, 
doi: http://dx.doi.org/10.15359/ree.21-1.14

URL: http://www.una.ac.cr/educare

CORREO: educare@una.cr

Pacheco y Villalobos, 2004; Villarreal, 2005). Estas se interpretan en la Tabla 2, con el objetivo específico, las categorías, las subcategorías y el instrumento por utilizar. Para cada categoría se establecieron las subcategorías correspondientes: buena líder, buen desempeño administrativo, mala administración, toma de decisiones, práctica directiva desde las visitas a las aulas, procesos de comunicación, valores y actitudes y relaciones humanas.

Tabla 2: Categorías de análisis

\begin{tabular}{|c|c|c|c|}
\hline $\begin{array}{l}\text { Propósito de la } \\
\text { investigación }\end{array}$ & Categorías & Subcategorías & Instrumento \\
\hline $\begin{array}{l}\text { Caracterizar el } \\
\text { desempeño de la } \\
\text { directora en el contexto } \\
\text { educativo desde la } \\
\text { mirada de los diferentes } \\
\text { actores involucrados en } \\
\text { el proceso. }\end{array}$ & $\begin{array}{l}\text { Conocimiento del } \\
\text { personal docente sobre } \\
\text { el desempeño de la } \\
\text { directora }\end{array}$ & $\begin{array}{l}\text { - Buena líder } \\
\text { - Buen desempeño administrativo } \\
\text { - Mala administración } \\
\text { - Toma de decisiones } \\
\text { - Práctica directiva } \\
\text { - Procesos de comunicación } \\
\text { - Valores y actitudes } \\
\text { - Relaciones humanas }\end{array}$ & $\begin{array}{l}\text { Entrevista } \\
\text { semiestructurada }\end{array}$ \\
\hline $\begin{array}{l}\text { Caracterizar el desempeño } \\
\text { de la directora en el } \\
\text { contexto educativo desde } \\
\text { la mirada de los diferentes } \\
\text { actores involucrados en el } \\
\text { proceso. }\end{array}$ & $\begin{array}{l}\text { Conocimiento del } \\
\text { estudiantado sobre } \\
\text { el desempeño de la } \\
\text { directora }\end{array}$ & $\begin{array}{l}\text { - Buena líder } \\
\text { - Toma de decisiones } \\
\text { - Práctica directiva } \\
\text { - Procesos de comunicación } \\
\text { - Valores y actitudes } \\
\text { - Buen desempeño administrativo } \\
\text { - Mala administración } \\
\text { - Relaciones humanas }\end{array}$ & Grupo focal \\
\hline $\begin{array}{l}\text { Caracterizar el desempeño } \\
\text { de la directora en el } \\
\text { contexto educativo desde } \\
\text { la mirada de los diferentes } \\
\text { actores involucrados en el } \\
\text { proceso. }\end{array}$ & $\begin{array}{l}\text { Conocimiento del padre } \\
\text { y la madre de familia } \\
\text { sobre el desempeño de } \\
\text { la directora }\end{array}$ & $\begin{array}{l}\text { - Buena líder } \\
\text { - Toma de decisiones } \\
\text { - Práctica directiva } \\
\text { - Procesos de comunicación } \\
\text { - Valores y actitudes } \\
\text { - Relaciones humanas } \\
\text { - Mala administración } \\
\text { - Buen desempeño administrativo }\end{array}$ & Grupo focal \\
\hline $\begin{array}{l}\text { Caracterizar el desempeño } \\
\text { de la directora en el } \\
\text { contexto educativo desde } \\
\text { la mirada de los diferentes } \\
\text { actores involucrados en el } \\
\text { proceso. }\end{array}$ & $\begin{array}{l}\text { Conocimiento del } \\
\text { personal administrativo } \\
\text { sobre el desempeño de } \\
\text { la directora }\end{array}$ & $\begin{array}{l}\text { - Buena líder } \\
\text { - Toma de decisiones } \\
\text { - Práctica directiva } \\
\text { - Procesos de comunicación } \\
\text { - Valores y actitudes } \\
\text { - Mala administración } \\
\text { - Relaciones humanas } \\
\text { - Buen desempeño administrativo }\end{array}$ & $\begin{array}{l}\text { Entrevista } \\
\text { semiestructurada }\end{array}$ \\
\hline
\end{tabular}

Nota: Elaboración propia. 
doi: http://dx.doi.org/10.15359/ree.21-1.14

URL: http://www.una.ac.cr/educare

CORREO: educare@una.cr

\section{Análisis de la información}

Se procede a elaborar un total de 11 entrevistas. La entrevista semiestructurada y el grupo focal fueron las técnicas empleadas para recopilar la información de las instituciones seleccionadas. Las conversaciones fueron grabadas; posteriormente se fueron transcribiendo una a una para luego iniciar con el correspondiente proceso del vaciado en la matriz donde se escribía la categoría de análisis con su respectiva subcategoría, el testimonio de la persona y, de esta forma, se fueron codificando los datos, la información se clasificó por colores, y se fue construyendo la matriz de análisis de información en la fase cualitativa.

Para el inicio de la triangulación de datos en la fase cualitativa se procedió, en primer lugar, a transcribir las entrevistas y los conversatorios. De esta manera, se detallan los testimonios descubiertos a través de la recopilación y triangulación de la información; en segundo lugar, ya con las entrevistas transcritas se procede a vaciar los testimonios de los participantes en una matriz donde se transcriben los argumentos en la casilla correspondiente y se validan, de acuerdo con las subcategorías que interesan trabajar; se etiquetan por colores para buscar, de esta manera, códigos importantes, así como triangular los testimonios de las personas entrevistadas, posteriormente, se procede a trabajar en el análisis de datos por medio de ATLASTI para vincular el análisis textual con las frases de contenido (expresión propia del programa ATLAS TI para denominar las categorías que va identificando) desde las categorías, subcategorías que interesan analizar, y códigos que se establecen con los testimonios y con ayuda de la matriz para triangular desde las voces participantes (ver Figura 2).

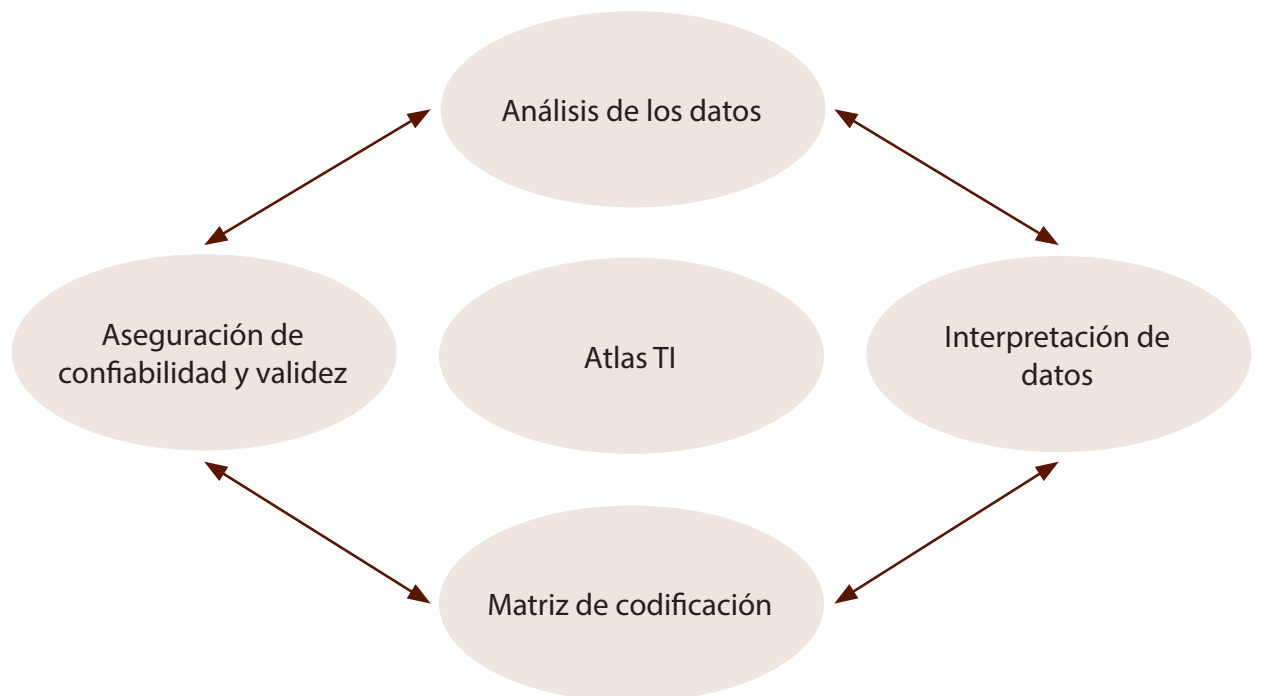

Figura 2: Análisis de datos de la fase cualitativa. 
doi: http://dx.doi.org/10.15359/ree.21-1.14

URL: http://www.una.ac.cr/educare

CORREO: educare@una.cr

\section{Interpretación y discusión de los resultados}

Se ha logrado demostrar que los miembros de la comunidad educativa valoran como muy positivo el desempeño profesional en la práctica directiva de ambas directoras graduadas del CIDE. Los testimonios mostraron, como referencia importante y desde la subcategoría buen desempeño administrativo de la directora, que el orden, la comunicación, el saber delegar, manejar las leyes, la responsabilidad, la organización, las decisiones en equipo, entre otras, son clave para el buen desempeño administrativo. Se presenta una síntesis de las entrevistas donde manifiestan cómo valoran el actuar de las directoras (lo que aparece entre paréntesis en la entrevista son los códigos que se utilizaron para llevar a cabo el análisis de la información de la investigación).

... el ambiente es diferente, las materias, el trato del docente al estudiante es muy distinto, entonces aquí los preparan para eso. \{P 9: E.P-CH (59:60)\}

... la calidad de la educación que les dan que es muy superior a las de otros colegios. \{P 9: E.P$\mathrm{CH}(28: 29)\}$

... se siente desde que ellos entran se preocupan por pequeños detalles. \{P 9: E.P-CH (34:35)\}

... En la mayoría de los casos considero que sí, siempre hay ciertas diferencias con respecto a lo que nosotros pensamos y lo que la administración piensa pero es cuestión de sentarse a conversar y resolver, lo que se trata es de ayudar a los estudiantes y buscar un consenso, principalmente en toma de decisiones o en aspectos que tienen que ver con el estudiantado. \{P 6:E.D3-CH(135:139)\}

... Si se toma una decisión, se es consecuente con eso, no se cede ante presiones, si se cambie tiene que suceder algo para que se cambie, hay coherencia. \{P 7:E.D4-CH (127:128)\}

Algunas de estas características fueron mencionadas reiteradamente por los grupos participantes, lo que concuerda con Latorre (2006) y Tello (2008) al manifestarlas como un buen desempeño administrativo.

Desde la opinión del estudiantado acerca del desempeño administrativo de la directora manifestaron como dato importante que una tarea es la de "saber controlar" para que se lleven a cabo las tareas institucionales, así como la "organización de los procesos", sobresale el hecho de brindarle apoyo al estudiantado, lo cual es visto como algo positivo, de igual manera, desde el aspecto humano ya que debe ser una persona "buena gente", y "buena líder".

Resulta interesante mencionar que el estudiantado opina que "el no dañar la institución", cuidándola, es otra forma de manifestar un buen desempeño administrativo. Se podría indicar 
doi: http://dx.doi.org/10.15359/ree.21-1.14

URL: http://www.una.ac.cr/educare

CORREO: educare@una.cr

que es importante para el estudiantado un espacio físico agradable, opinión reiterativa. Se evidencia que el espacio físico incide en la motivación, así como en el aprendizaje y eso es algo que se reafirma en el Tercer informe del estado de la educación (Programa Estado de la Nación, 2011). Cabe mencionar, además, que en ese mismo Informe, particularmente, en la investigación de Sánchez, Francis y Alfaro (2011), la gestión de calidad se entiende como "un proceso de planificación, seguimiento, evaluación y mejoramiento permanente a la luz de la misión, visión y lo objetivos de la estructura organizativa" (p. 337).

Se evidencia en los hallazgos de la presente investigación que una gestión de calidad debe ser ante todo un proceso de participación, trabajo en equipo, comunicación y acercamiento hacia las personas desde la dirección; eso es evidente y coincide plenamente con las unidades de contenido de la investigación propuesta. Se puede destacar, de igual manera, que las relaciones interpersonales desarrolladas por miembros de la organización, entendidas como las relaciones humanas, son vitales para un sano y apropiado manejo institucional; se espera que el clima laboral sea de crecimiento personal y colectivo; la confianza, el respeto, la cordialidad son ingredientes del día a día, sin olvidar que el sentirse parte de un colectivo favorece las relaciones tal y como se menciona en las entrevistas:

El clima laboral es excelente como en todo siempre hay alguien que no está de acuerdo, o que no se lleva bien con el resto, las relaciones son muy buenas entre todos. \{P7:E.D4-CH (164:166)\}

... hay muy buen ambiente de trabajo, los caracteres, respeto, organización, una buena administración, un proyecto a cumplir. \{P12:E.D.5-CH(188:190)\}

La libertad que tengo para trabajar aquí no la tengo en otros lugares ... \{P6:E.D3-CH(180:184)\}

Siempre un ambiente vacilón, ambiente muy bonito, me ha gustado demasiado, bastante. \{P3:E.D.6-CH (162:163)\}

Son excelentes porque hay compañeros que uno comparte en los consejos, muchos trabajamos en otras instituciones y los compañeros que llegan nuevos se adaptan ... \{P6:E. D3-CH (177:179)\}

Yo me siento muy contenta de estar aquí. Yo he trabajado en otros colegios y eso es importante, y uno aprende. Siempre es para mejorar. \{P12: E.D.5-CH (193:194)\}

... el trabajo aquí es en equipo. \{P4:E.D1-ESJ (126:126)\} 
doi: http://dx.doi.org/10.15359/ree.21-1.14

URL: http://www.una.ac.cr/educare

CORREO: educare@una.cr

... el trato siempre habla y conversa con los estudiantes antes de aplicar una boleta"... \{P5:E. D2-ESJ (121:122)\}

... hay mucho respeto y ella se lo ha ganado como autoridad, el personal tiene mucho respeto. \{P4:E.D1-ESJ (124:125)\}

... lo más importante es que a uno lo traten bien y aquí hay eso, ella anda supervisando en los recreos. \{P4:E.D1-ESJ (127:129)\}

... respeto, atenta a escucharlo a uno, reconoce el buen trabajo que se lleva a cabo, los agradecimientos en cada actividad. \{P5: E.D2-ESJ (129:130)\}

Además el liderazgo tiene que ver con respeto, confianza, el transmitir seguridad y saber transmitirlo, exigir pero con cariño, la directora es el eje principal y así se evidencia en las entrevistas:

... ella es el eje principal, ella tiene que articular de un forma adecuada el trabajo de los docentes, con los estudiantes. \{P 9: E.P-CH(74:75)\}

... para mí es una buena líder. $\{P$ 9: E.P-CH(82:82)\}

... digamos como líder, o sea ella mantiene su liderazgo, su respeto, pero sin, como dice la señora, sin hacerse sentir como de más, como que hay una confianza, una relación muy linda entre ellos. $\{$ P 9: E.P-CH(90:92)\}

... a ella se le respeta como lo que es, como la directora. $\{P$ 9: E.P-CH(94:94)\}

Y ella transmite como mucha seguridad, o sea ella es muy segura de sí misma y lo transmite; lo sabe transmitir. \{P 9: E.P-CH(98:99)\}

Yo siento que exige pero con cariño. \{P 9: E.P-CH (103:103)\}

La comunicación es otro elemento fundamental dentro del proceso administrativo y, según el análisis efectuado, es aquel que permite intercambiar información, trabajo, procedimientos donde existe claridad tanto de lo que se hace como de lo que se dice, de este modo las directoras deben ser consecuentes con lo que se dice tanto como con lo que se hace. No se puede olvidar que la comunicación debe ser vista como una herramienta poderosa para el éxito y el crecimiento de las personas que integran la organización y, en este caso, la prioridad que le otorguen los entes directivos escolares, pues de eso depende en buena parte su buen desempeño administrativo, tal y como queda demostrado en el análisis de los resultados: 
doi: http://dx.doi.org/10.15359/ree.21-1.14

URL: http://www.una.ac.cr/educare

CORREO: educare@una.cr

... cada mes hay consejo y actividad planeada, da tiempo a ordenarse, hay posibilidad de orden, si hay algún tema en particular hablar con la directora. Con ella siempre me ha ido bien. $\{P$ 3: E.D.6-CH (83:85)\}

... la comunicación con todos los que intervienen en todo este proceso. \{P 6:E.D3-CH(105:106)\}

... la facilidad de que siempre podemos conversar con ella... \{P12: E.D.5-CH(72:73)\}

... primero la comunicación por correos se nos informan las cosas. \{P 3: E.D.6-CH(50:51)\}

... siempre hay ciertas diferencias con respecto a lo que nosotros pensamos y lo que la administración piensa pero es cuestión de sentarse a conversar y resolver. \{P6: E.D3-CH (135:137)\}

... siempre se me ha comunicado todo. \{P 3:E.D.6-CH (27:27)\}

... hay con ella una buena comunicación, no es todo perfecto pero vamos creciendo en el proceso. $\{$ P12: E.D.5-CH (119:120)\}

Buena comunicación, buen trato, hay facilidad para conversar cuando algo no parece o no funciona como se quiere... \{P12: E.D.5-CH (49:50)\}

Según Borrel, citado por Braslavsky et al. (2004), presentar la información en forma concreta y clara, dar a conocer la información que afecta a los diversos actores, seleccionar un sistema ágil y eficaz para transmitirla, establecer canales claros de comunicación para que esta circule son aspectos que caracterizan un centro escolar sano, con buenos sistemas de comunicación que permitan contar con suficiente información para detectar y comprender el origen de posibles ejes de tensión, para luego resolver, y así, a partir de esta perspectiva, consolidar el movimiento de mejora escolar.

Es importante, desde la institución escolar, comprender su funcionamiento e incidir en su mejora a través de mecanismos que integran a todos los sujetos involucrados en espacios para intercambiar información útil para la acción y eso es evidente desde los resultados de la indagación, Cuarto informe del estado de la educación (Programa Estado dela Nación, 2013). Dentro del proceso de interpretación de los resultados en la dimensión de buena líder, el sentir de las personas encuestadas e informantes es que ambas directoras tienen características personales que las hacen percibir como líderes de la institución respectiva, hay una plena identificación y confianza en su quehacer, lo que evidencia que su influencia es aceptada legítimamente. 
doi: http://dx.doi.org/10.15359/ree.21-1.14

URL: http://www.una.ac.cr/educare

CORREO: educare@una.cr

El Centro de Estudios de Política en Educación, CEPPE (2009) afirma que:

La relevancia que adquiere el liderazgo directivo radica en que la presencia de ciertas prácticas de dirección escolar tiene un impacto positivo en las condiciones y trabajo de los docentes y, consecuentemente, en los resultados de aprendizaje de las escuelas. (p. 20)

Sin embargo, estos autores mencionan que un deficiente liderazgo por parte de quien ocupe la dirección lleva a disminuir el aprendizaje del estudiantado, a retroceder a las escuelas en calidad y, tal y como se abordó en el estudio, se podría mencionar que eso ocasionaría mala administración institucional. Por lo tanto, se podría afirmar y así lo demuestran los hallazgos de los testimonios, que existe un consenso en cuanto a que el liderazgo está vinculado con la práctica de mejora educativa, así como un buen grado de avance en el trabajo colaborativo, la comunicación, la toma de decisiones, el saber delegar, entre otros aspectos.

Sí, ella tiene el papel claro que muchas veces tiene que seguir una línea, mucho potencial en eso que es liderazgo. \{P10: E.PAmd-CH(26:27)\}

... en consejo ella siempre presenta algo, y todos la siguen, aunque acepta opiniones pero sigue la línea que ella sigue. \{P10: E.PAmd-CH(28:30)\}

La gente se fue encauzando a una línea mejor \{P10: E.PAmd-CH(32:32)\}

... tiene facilidad de palabra el trabajo en equipo. \{P10: E.PAmd-CH(153:153)\}

Barber y Mourshed, (2008) señalan la vinculación que existe entre el liderazgo y los sistemas educativos exitosos que, de alguna manera, se relaciona con el estudio llevado a cabo según opinión de los sujetos entrevistados:

Boston, Inglaterra y Singapur demuestran que un buen liderazgo escolar es esencial para poner en práctica cambios sustanciales y rápidos. Los sistemas educativos con más alto desempeño cuentan con un conocimiento sustancial y creciente acerca de qué constituye un liderazgo escolar efectivo para convertir a sus directores en impulsores de mejoras en la instrucción. (p. 33)

En ese sentido, Sánchez et al. (2011) brindan algunos parámetros que determinan la gestión de calidad en las instituciones escolares e interesa señalar el de liderazgo directivo: la orientación de la dirección promueve una visión y metas claras, delega tareas, responsabilidades, desarrolla acciones efectivas con la ayuda de la comunicación para la comunidad educativa, 
doi: http://dx.doi.org/10.15359/ree.21-1.14

URL: http://www.una.ac.cr/educare

CORREO: educare@una.cr

mencionan el clima institucional donde se promueve un estilo conciliador, así como promueve las capacidades y potencialidades de docentes y estudiantes. $Y$ esto es coincidente con los hallazgos de la investigación desde las voces participantes.

Quéella, cuando tiene un problema le gusta hablar antes de hacer las cosas. \{P 8:E.E-ESJ(84:85)\}

Ella es muy buena gente, muy inteligente, le gusta mucho dialogar y compartir actividades con los niños. \{P 8: E.E-ESJ (98:99)\}

Es así como se ha querido tratar de explorar el significado de las experiencias de las personas involucradas en el estudio, describir a partir de su vida cotidiana y desde lo que compete a sus prácticas como integrantes de un colectivo. Rendón, citada por Rhem (2009), ha iniciado un debate académico concerniente a que los sentimientos, los pensamientos y la pedagogía son elementos integrales, así como que los valores tales como la justicia y la liberación deben ser abordadas por las personas encargadas de dirigir las instituciones escolares. Se menciona lo anterior, ya que según los hallazgos aquí encontrados también se deben hacer esfuerzos para mejorar aún más esos aspectos.

\section{A manera de conclusión}

- El desempeño de las directoras en el contexto educativo, desde la mirada de la comunidad educativa. Se reconoce el papel que ambas realizan, especialmente, en lo que el ejercicio del liderazgo, procesos de comunicación, toma de decisiones, relaciones humanas, la buena administración y su práctica directiva.

- Una mala administración, según las personas participantes del estudio, es vista como algo que ocasionaría desorden, movilidad constante del profesorado, desmotivación por parte de las personas integrantes de la comunidad educativa, bajo rendimiento, en otras palabras, caos institucional y eso es coincidente con la teoría estudiada.

- La buena administración obedece a un apropiado manejo de las relaciones humanas, especialmente, desde la mirada de los diferentes actores involucrados en la investigación, ya que de eso depende en buena parte la marcha de la institución, la cordialidad, la motivación, la buena comunicación, el saber delegar, escuchar, ser humilde son aspectos esenciales.

- El liderazgo en una institución educativa es socialmente construido y se manifiesta en la institución escolar y eso se demostró en el análisis efectuado, tanto por miembros de la comunidad educativa como por quienes ocupan la dirección. 
doi: http://dx.doi.org/10.15359/ree.21-1.14

URL: http://www.una.ac.cr/educare

CORREO: educare@una.cr

- Se asigna una importancia cada vez mayor al papel que desempeña las personas que ocupan cargos de dirección escolar donde destaca que el ejercicio del liderazgo es especialmente colectivo y no aparece como desarticulado e individualizado. Eso la teoría lo respalda.

- Pareciera que, para lograr una escuela exitosa, se requiere de un compromiso conjunto desde el papel del personal directivo, el equipo docente, la comunidad educativa, donde se gestiona el aprendizaje y la enseñanza para lograr desarrollar en las personas su potencialidad.

- El desarrollo de las personas en las organizaciones educativas, especialmente el cuerpo docente, es una tarea de la persona encargada de dirigir la institución donde la actitud de confianza hacia estas personas, hacia sus capacidades, e intereses resulta de especial interés. Esto es coincidente con la teoría de la investigación.

- El proceso de toma de decisiones y la comunicación desde las instituciones educativas participantes demostró articulación, flexibilidad, diálogo respetuoso, comprensión, humildad entendida como la capacidad para relacionarse con las demás personas de forma cercana y empática, los que constituyen ingredientes básicos para la buena convivencia institucional.

Es así como, para finalizar con este análisis, se hace necesario resaltar una posible indagación que podría surgir. Esta es la correspondiente a los aspectos concernientes a las características personales de quienes ejercen el papel de director o directora escolar a partir de sus motivaciones, valores y modos en relación con la comunidad escolar.

\section{Referencias}

Barber, M. y Moushed, M. (2008). ¿Cómo hicieron los sistemas educativos con mejor desempeño del mundo para alcanzar sus objetivos? PREAL, 41, 1-48. Recuperado de http://www.oei. es/noticias/spip.php?article3077

Barrera-Osorio, F., Fasih, T., Patrinos, H. A. y Santibáñez, L. (2009). Decentralized decision-making in schools. The theory and evidence on school-based management. Washington, DC.: TheWorld Bank. doi: https://doi.org/10.1596/978-0-8213-7969-1

Bernardini, A. (2003). Educación para la paz [videoconferencia]. Programa de Doctorado de la UNED, San José, Costa Rica. 
doi: http://dx.doi.org/10.15359/ree.21-1.14

URL: http://www.una.ac.cr/educare

CORREO: educare@una.cr

Braslavsky, C., Acosta, F. y Jabif, L. (2004). Directores en acción. Módulos de formación en competencias para la gestión escolar en contextos de pobreza. Orientaciones conceptuales y didácticas. Buenos Aires: IIPE-Unesco. Recuperado de http://www.buenosaires.iipe. unesco.org/sites/default/files/Modulos\%20para\%20la\%20formacion $\% 20$ en 20 competencias\%20para\%20la\%20gestion\%20escolar\%20en\%20cont\%20pob.pdf

Cano, J. S. (2009). Evolución de la administración y la gestión escolar. Tendencias Pedagógicas, 14, 159-168. Recuperado de http://dialnet.unirioja.es/servlet/articulo?codigo=3002738

Centro de Estudios de Política en Educación (CEPPE). (2009). ¿Prácticas de liderazgo directivo y resultados de aprendizaje hacia conceptos capaces de guiar la investigación empírica. REICE. Revista Iberoamericana sobre calidad, eficacia y cambio en Educación, 7(3), 19-33. Recuperado de http://www.rinace.net/reice/numeros/arts/vol7num3/art2.pdf

Concha, C. (2007). Claves para la formación de directivos de instituciones escolares. REICE Revista Electrónica Iberoamericana sobre calidad, eficacia y cambio en Educación, 5(5), 133138. Recuperado de http://dialnet.unirioja.es/servlet/articulo?codigo $=2515079$

Coronel, J. M. (2005). El liderazgo del profesorado en las organizaciones educativas: Temáticas para su análisis e investigación. Revista Española de Pedagogía, 63(232), 471-490.

Cuenca, R. (19 de abril, 2012). Las múltiples identidades profesionales de la docencia (Video]. Conferencia presentada en el III Congreso Pedagógico "Formación profesional del educador y la educadora: Una responsabilidad Compartida". San José, Costa Rica: COLYPRO. Recuperado de https://www.youtube.com/watch?v=E2MvPXXXR g

Flick, U. (2007). Introducción a la investigación cualitativa (2a ed.). Madrid: Morata.

Garbanzo, G. M. y Orozco, V. H. (2010). Liderazgo para una gestión moderna de procesos educativos. Revista Educación, 34(1), 15-29. doi: https://doi.org/10.15517/revedu.v34i1.495

Gurdián-Fernández, A. (2010). El paradigma cualitativo en la investigación socio-educativa. San José, Costa Rica: EUCR. Recuperado de http://web.ua.es/en/ice/documentos/recursos/ materiales/el-paradigma-cualitativo-en-la-investigacion-socio-educativa.pdf

Hernández, R., Fernández, C., y Baptista, P. (2010). Metodología de la investigación (5ª ed.). México: Mc Graw-Hill.

Latorre, M. (2006). Nuevas miradas, viejos problemas: Las relaciones entre formación inicial y ejercicio profesional docente. Foro Educacional, 10, 41-63. Recuperado de https://dialnet. unirioja.es/servlet/articulo?codigo $=2292721$ 
doi: http://dx.doi.org/10.15359/ree.21-1.14

URL: http://www.una.ac.cr/educare

CORREO: educare@una.cr

Organización de Estados Iberoamericanos para la Educación, la Ciencia y la Cultura (OEI). (2010). 2021 metas educativas. La educación que queremos para la generación de los bicentenarios. Madrid: Autor. Recuperado de http://www.oei.es/metas2021/libro.htm

Pareja, J. A. (2009).Liderazgo y conflicto en las organizaciones educativas. Educación y Educadores, 12(1), 137-152. Recuperado de http://www.redalyc.org/articulo.oa?id=83411512010

Pozner, P. (2000). Módulo 7. Resolución de problemas. Diez módulos destinados a los responsables de los procesos de transformación educativa. En P. Pozner (Autora), Competencias para la profesionalizacióln de la gestión educativa (pp. 1-36). Buenos Aires: IIPE-Unesco. Recuperado de http://www.colombiaaprende.edu.co/html/home/1592/articles-189023 archivo 7.pdf

Programa Estado de la Nación. (2011). Tercer informe estado de la educación. San José, Costa Rica: Autor. Recuperado de http://www.estadonacion.or.cr/informe-iii-estado-educacion

Programa Estado de la Nación. (2013). Cuarto informe estado de la educación. San José, Costa Rica: Autor. Recuperado de http://www.estadonacion.or.cr/informe-iv-estado-educacion

Rhem, J. (Ed.). (2009). Sentipensante Pedagogy. The National Teaching \& Learning Forum, 18(2), 1-4.

Sánchez, V., Francis, S. y Alfaro, G. (2011). Estudio 4. Cultura organizacional de los colegios desde la óptica de las y los profesores. En Programa Estado de la Nación (Autor), Tercer informe estado de la educación (pp. 335-347). San José, Costa Rica: Autor. Recuperado de http:// www.estadonacion.or.cr/informe-iii-estado-educacion

Sandín, M. P. (2003). Investigación cualitativa en educación. Fundamentos y tradiciones. Madrid: McGraw-Hill.

Tello, C. G. (2008). Gestionar la escuela en Latinoamérica. Gestión educativa, realidad y política. Revista Iberoamericana de Educación, 45(6) 1-10. Recuperado de http://www.rieoei. org/2367.htm

Vargas, I. (2004). La inteligencia emocional en las instituciones educativas. Revista Electrónica Educare, 7, 107-114. Recuperado de http://www.revistas.una.ac.cr/index.php/EDUCARE/ issue/view/185/showToc

Vargas, I. (Noviembre, 2007). Perspectivas y desafíos de la administración educativa. Ponencia presentada en el III Congreso Internacional de Administración de la Educación. Congreso llevado a cabo en la Universidad de Costa Rica, San José, Costa Rica.

Vargas, I. (Noviembre, 2010). Gestión e innovación en contextos de aprendizaje: Reflexiones 
doi: http://dx.doi.org/10.15359/ree.21-1.14

URL: http://www.una.ac.cr/educare

y desafíos. Ponencia presentada en el IV Congreso Internacional de Administración de la Educación. Gestión, Innovación, Inclusión y Gobernabilidad en contextos educativos complejos 2010. Congreso llevado a cabo en la Universidad de Costa Rica, San José, Costa Rica.

Vargas, I. (2014). La formación profesional de directoras y directores graduados de la carrera de Administración Educativa del Centro de Investigación y Docencia en Educación de la Universidad Nacional: Análisis de su práctica directiva en dos instituciones educativas (Tesis doctoral). Universidad Estatal a Distancia. San José, Costa Rica.

Vargas, I., Pacheco, V. y Villalobos, E. (2004). Plan de rediseño de la carrera de licenciatura en administración educativa. Heredia, Costa Rica: Universidad Nacional, CIDE, División de Educación para el Trabajo.

Villarreal, E. (2005). La efectividad de la gestión escolar depende de la formación del recurso humano como factor, actor, y promotor del cambio dentro de los procesos, dimensiones y políticas educativas. Revista Iberoamericana de Educación, 37(2), 1-4. Recuperado de http://rieoei.org/1083.htm 